\title{
Comparative Evaluation of Elliptic Curve Cryptography Based Homomorphic Encryption Schemes for a Novel Secure Multiparty Computation
}

\author{
Sankita J. Patel, Ankit Chouhan, Devesh C. Jinwala \\ Department of Computer Engineering, S V National Institute of Technology, Surat, India \\ Email: sankitapatel@gmail.com, chouhan.ankit03@gmail.com, dcjinwala@gmail.com
}

Received November 30, 2013; revised December 30, 2013; accepted January 6, 2014

Copyright (C) 2014 Sankita J. Patel et al. This is an open access article distributed under the Creative Commons Attribution License, which permits unrestricted use, distribution, and reproduction in any medium, provided the original work is properly cited. In accordance of the Creative Commons Attribution License all Copyrights @ 2014 are reserved for SCIRP and the owner of the intellectual property Sankita J. Patel et al. All Copyright (C) 2014 are guarded by law and by SCIRP as a guardian.

\begin{abstract}
In this paper, we focus on Elliptic Curve Cryptography based approach for Secure Multiparty Computation (SMC) problem. Widespread proliferation of data and the growth of communication technologies have enabled collaborative computations among parties in distributed scenario. Preserving privacy of data owned by parties is crucial in such scenarios. Classical approach to SMC is to perform computation using Trusted Third Party (TTP). However, in practical scenario, TTPs are hard to achieve and it is imperative to eliminate TTP in SMC. In addition, existing solutions proposed for SMC use classical homomorphic encryption schemes such as RSA and Paillier. Due to the higher cost incurred by such cryptosystems, the resultant SMC protocols are not scalable. We propose Elliptic Curve Cryptography (ECC) based approach for SMC that is scalable in terms of computational and communication cost and avoids TTP. In literature, there do exist various ECC based homomorphic schemes and it is imperative to investigate and analyze these schemes in order to select the suitable for a given application. In this paper, we empirically analyze various ECC based homomorphic encryption schemes based on performance metrics such as computational cost and communication cost. We recommend an efficient algorithm amongst several selected ones, that offers security with lesser overheads and can be applied in any application demanding privacy.
\end{abstract}

\section{KEYWORDS}

Elliptic Curve Cryptography; Privacy Preservation; Secure Multiparty Computation

\section{Introduction}

Our handhelds have become smaller; computers faster; disks larger; networks more efficient and we enjoy bandwidths like never before; everything grew exponentially. All this sums up to a very favourable environment for data collection, transfer and storage. In order to fully utilize this data, there is a need to perform collaborative computation on data. However, the data collected mostly contain information related to individuals, their financial status, lifestyle and social behaviour in general. Joint computation on data may pose threat to privacy of individual's data. Hence, there is a need to protocol a device that performs joint computation on private data without revealing data to other parties. Secure Multiparty Com- putation (SMC) addresses this issue. The general framework for SMC consists of specifying a random process that maps $\mathrm{m}$ inputs (local inputs of parties) to m outputs (desired outputs) [1]. The random process describes desired functionality. We focus on addition function in this paper.

There are many real world scenarios where privacy can be an issue, a few of which are worth mentioning: Considering the field of medical research, considering the case that a number of different hospitals wish to perform joint research on their patient data. Also, let us assume that privacy policy and law prevent these hospitals from over pooling their data or revealing it to each other, due to the confidentiality of patient records. In such cases, it is necessary to find a solution that enables the hospitals 
to compute the desired functionality on the union of their databases, without ever pooling or revealing their data.

Consider the interaction between different intelligence agencies; for security purposes, these agencies cannot allow each other free access to their confidential information; if they did, then a single mole in a single agency would have access to an overwhelming number of sources. It is much more likely that suspicious behaviour would be detected if different agencies were able to perform computations on their combined data [2].

One way to compute the desired functionality is to use Trusted Third Party (TTP). In this scenario, parties send their data to TTP and TTP then computes results on parties' data and sends the output to all parties. However, the pivotal question in cryptography is to achieve TTPs that are indeed trusted. This demands protocols that eliminate TTP. In this paper, we propose a protocol that eliminates TTP.

There are three approaches to performing desired functionality in Secure Multiparty Computation viz. the Oblivious Transfer [3] Protocol, the homomorphic encryption [4] and the secret sharing [5]. The oblivious transfer protocol is costly in terms of computational and communication overheads. The secret sharing based approach gives better results in terms of computational cost due to primitive operations involved [6]. However, it requires existence of private channels. In addition, as pointed out in [7], the communication cost is higher due to message exchange between other parties in the protocol as explained in Section 3. The homomorphic encryption based approach does not require existence of private channel and assures high level of privacy. Hence, we focus on homomorphic encryption based approach in this paper. The homomorphic encryption is introduced in Section 2.

In literature, there do exist approaches that perform secure multiparty computation using homomorphic encryption. However, they use classical encryption schemes [8,9]. Elliptic Curve Cryptography (ECC) based approach gives promising results as classical encryption schemes $[10,11]$. This is due to the higher per bit security provided by ECC. To give an example, to provide equivalent security of 1024-bit RSA, an ECC scheme needs 160 bits parameters [12]. Hence, in this paper, we utilize ECC based approach to implementing secure multiparty computation. The approach is described in Section 3. We investigate various additively homomorphic encryptions schemes and analyze them empirically in Section 4.

A few approaches utilize ECC based encryption schemes. However, they require multiple encryptiondecryption at each site and hence are computationally expensive $[13,14]$. In our approach, we avoid multiple cipher operation at each site and thus reduce the computational cost of SMC. We justify this in Section 3.

\section{Background and Related Work}

The multi-party computation problem was introduced by Yao [15] and extended by Goldreich, Micali and Wigderson [16]. The basic method they use is to represent the problem as combinatorial circuit. Participating parties then run a protocol for every gate in the circuit. Though general and simple, the approach exhibits combinatorial explosion in terms of circuit size and hence not scalable for large inputs. Apart from scrambled circuit, Goldreich [1] presents various other methods to perform multiparty computation such as homomorphic encryption and secret sharing. In literature, various applications such as Privacy Preserving Data Mining [17,18], Private statistical information retrieval $[19,20]$, Privacy Preserving Database access [21] have been proposed that demand Secure Multiparty Computation among parties.

In Privacy Preserving Data Mining, various approaches for classification [22], clustering [23] and association rule mining have been proposed [24]. The basic building blocks in these approaches are private value addition and comparison. In this paper, we focus on private value addition. To give an example, in privacy preserving distributed clustering implements weighted average problem(WAP) as a basic building block [25]. Parties first perform local clustering at each site and then global cluster means are calculated using WAP. For example, consider two party scenario with party A and B. The sum of records and number of records for single cluster after computing local cluster means at party A and B is $\operatorname{sum}_{\mathrm{A}}$, $\mathrm{n}_{\mathrm{A}}$ and $\operatorname{sum}_{\mathrm{B}}, \mathrm{n}_{\mathrm{B}}$ respectively. Global means are computed using $\left(\operatorname{sum}_{A}+\operatorname{sum}_{B}\right) /\left(n_{A}+n_{B}\right)$. This requires the global addition of private value as a basic operation. Division is then carried out by parties locally [7].

In Wireless Sensor Network, the in-network processing demands the privacy. The in-network processing refers to on-the-fly processing of data packets by intermediate nodes before being communicated them to the base station. However, to protect the privacy of individual sensor reading, this on-the-fly processing must be done in a privacy preserving way. This process uses notion similar to multiparty computation. However, the computation is carried out at aggregator node only and not at all the nodes. This process utilizes homomorphic encryption schemes to be implemented at intermediate nodes.

\subsection{Homomorphic Encryption}

Homomorphic Encryption schemes allow parties to perform simple computations on encrypted data. As the computations are performed on encrypted data, the data is not revealed in the process and the privacy is preserved. Typically, a third party can calculate one of the encrypted sum or the encrypted product of two encrypted messages. This makes homomorphic cryptosystems useful in secure 
multiparty computation and a wide variety of privacy preserving protocols.

The additive homomorphic algorithm whose input is the public key of the encryption scheme and two ciphertexts, and whose output is

$E_{p_{k}}\left(m_{1}\right)+_{p_{k}} E_{p_{k}}\left(m_{2}\right)=E_{p_{k}}\left(m_{1}+m_{2}\right)$; where $+_{p_{k}}$ is the homomorphic addition function, $E_{p_{k}}$ is the publickey encryption function and $m_{1}$ and $m_{2}$ are elements in the domain of data. We refer additively homomorphic encryption scheme based on ECC in this paper.

\subsection{Elliptic Curve Cryptography (ECC)}

ECC is a public key cryptography approach based on the algebraic structure of elliptic curves over finite fields $[10,11]$. There are two types of finite fields where the elliptic curves are defined: prime fields $F_{p}$, where $\mathrm{p}$ is a large prime number, and binary fields $F_{2}^{m}$. In this work, we are interested in the use of elliptic curves over prime fields $E\left(F_{p}\right)$. A nonsupersingular elliptic curve $E$ over $F_{p}$ is defined as the solution of $(x, y) \in F_{p} \times F_{p}$ to the cubic equation:

$$
y^{2}=x^{3}+\mathrm{ax}+\mathrm{b} \bmod \mathrm{p}
$$

where $a, \quad b \in F_{p}$ such that $4 a^{3}+27 b^{2} \neq 0(\bmod p)$ together with a special point $\infty$ called the point at infinity, The group of points forms an abelian group with addition operation so that the addition of any two points results in another point on the same curve. The security of ECC based cryptographic protocol is based on the Elliptic Curve Discrete Logarithm Problem (ECDLP). The ECDLP can be defined as the problem of finding the scalar $\mathrm{k}$ such that $\mathrm{Q}=\mathrm{kP}$ given $\mathrm{Q}$ and $\mathrm{P}$ (generator point).

\subsection{Algorithms Used for Evaluation}

We utilize additively homomorphic encryption schemes for evaluation. We investigate four algorithms that are additively homomorphic. They are Elliptic Curve Naccache-Stern (EC-NS) Encryption [26], Elliptic Curve Okamoto-Uchiyama (EC-OU) Encryption [27], Elliptic Curve Paillier (EC-P) Encryption [28] and Elliptic Curve Elgamal(EC-EG) Encryption [29]. First three schemes are described in [30] and are Elliptic Curve variants of the previously proposed schemes by Naccache-Stern [26], Okamoto-Uchiyama [27] and Pailler [28]. EC-EG is the variant of ElGamal [29]. Original ElGamal is multiplicatively homomorphic while EC-EG is transformed to additive group and hence is additively homomorphic. The pseudo code and other details may be found in [30].

\section{The Proposed Approach: Secure Multiparty Computation Using ECC}

We propose novel approach to Secure Multiparty Addition problem using ECC. Secure Multiparty Addition have beem implemented for privacy preserving data mining using homomorphic encryption [25] and secret sharing [7]. However, the approach of [25] uses classical public key encryption scheme and hence is computationally expensive. Further, multiple cipher operations at each site increased the computational cost. The secret sharing based approach proposed in [7] is efficient in terms of computational cost. However, due to two rounds of information exchange among parties increases the communication cost. Among the three approaches to implement SMC viz. the oblivious transfer based, the homomorphic encryption based and the secret sharing based, we focus on homomorphic encryption based approach.

We allow parties to communicate in ring topology. Consider three party scenario with parties A, B and C with private values $m_{1}, m_{2}$ and $m_{3}$ respectively. Parties need to compute $m_{1}+m_{2}+m_{3}$ securely. One party in the protocol is randomly designated as the Initiator party. The proposed approach considering party A as the Initiator is shown in Figure 1.

As shown in Figure 1, the initiator first encrypts its private value using ECC based encryption scheme. The resultant ciphertext (which is in the form of elliptic curve point) is sent to next party in the ring. Next party does not perform any cipher operation but just adds its own private value (mapped to elliptic curve point) with the received cipher text. This process is repeated and finally initiator receives the message $\mathrm{E}\left(\mathrm{m}_{1}\right)+\mathrm{m}_{2}+\mathrm{m}_{3}$ at the end of phase I. In phase II, initiator decrypts the message by removing the noise (that was added during encryption) from the message and computes $m_{1+} m_{2}+m_{3}$. Here, initiator just removes the noise in order to get desired sum. Hence, we have slightly modified the decryption processes of algorithms to get the sum value. This sum is then sent to next party in the ring and eventually all parties will receive the sum.

\section{Theoretical Analysis}

In this section, we discuss the computational and communication cost of our proposed approach.

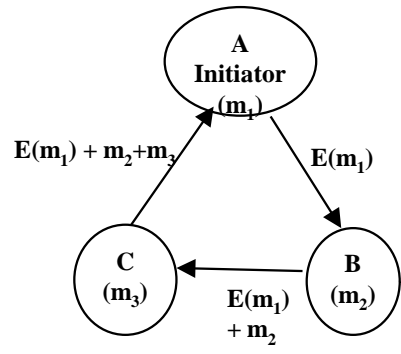

PhaseI

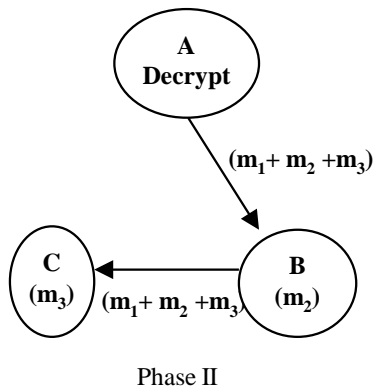

Phase II
Figure 1. Secure Multiparty Addition using Elliptic Curve Cryptography. 


\subsection{Computational Cost}

The computational cost is mainly due to the operations in first phase where parties send data to the next party in ring. However, we need to consider the cost for initiator and the rest of the parties separately. This is because the initiator encrypts and decrypts the data and the rest of the parties just need to add their data in the received data. Suppose to encrypt the data, time taken is T1. It is pointed out in [10] that by means of O(logk) addition and doubling, one can compute k. M value. Hence, T1 becomes $\mathrm{O}(\operatorname{logk})$. Further, to decrypt the data, time taken is T2. Let us have the time taken by other parties to add their data point is T3. Hence, the computation cost for initiator can be $=(\mathrm{O}(\operatorname{logk})+\mathrm{T} 2)=\mathrm{O}(\log \mathrm{k})$. For the rest of the parties the cost becomes $\mathrm{T} 3$ and hence $\mathrm{O}(1)$. For total of $\mathrm{N}$ parties the cost becomes $\mathrm{O}(\operatorname{logk})+\mathrm{O}(\mathrm{N})$.

\subsection{Communication Cost}

Our proposed approach involves two phases. In first phase, single message (as elliptic curve points) is communicated by each party to the next party in the ring. Hence, total $\mathrm{N}$ messages are transmitted for $\mathrm{N}$ party scenario. In second phase, only the sum (that is calculated) by initiator party is communicated to all parties in a ring. The last party in the ring need not send this sum to initiator. Hence, total (N-1) messages are transmitted in second round. Thus for $\mathrm{N}$ parties in the protocol, the communication cost becomes $\mathrm{O}(\mathrm{N})$ i.e. linear in terms of number of parties.

\subsection{Comparison with Secret Sharing Based Approach}

Our proposed approach is closest to the approach of [7]. In [7], privacy preserving clustering protocol is proposed using secret sharing scheme. The basic building block they use is the secure multiparty addition. Hence, we compare our approach with the approach of [7]. The approach of [7] is efficient in terms of computational cost due to primitive operations required in secret sharing scheme. However, approach of [7] requires message exchange among every other party in the protocol. Table 1 shows the comparison in communication cost. We achieve $\mathrm{O}(\mathrm{N})$ complexity with respect to communication cost as compared to $\mathrm{O}\left(\mathrm{N}^{2}\right)$ of secret sharing based approach. Hence, our approach achieves scalability with respect to number of parties in the distributed scenario.

Table 1. Comparison with respect to communication cost between our approach and approach of [7].

\begin{tabular}{cccc}
\hline & Phase I & Phase II & Total Complexity \\
\hline Our approach & $\mathrm{N}$ & $(\mathrm{N}-1)$ & 2N-1, hence $\mathrm{O}(\mathrm{N})$ \\
Approach of [7] & $\mathrm{N}(\mathrm{N}-1)$ & $\mathrm{N}(\mathrm{N}-1)$ & 2N(N-1), hence $\mathrm{O}\left(\mathrm{N}^{2}\right)$ \\
\hline
\end{tabular}

\section{Experimental Results}

In this paper, we focus on comparative evaluation of ECC based homomorphic encryption schemes to implement secure multiparty addition. We implement our proposed secure multi party addition protocol using four ECC based schemes such as EC-NS, EC-OU, EC-P and EC-EG.

\subsection{Experimental Setup}

We carry out the experiments in JAVA. The experiments are conducted on three different machines to emulate the true distributed scenario consisting of three parties. All machines have similar configuration of Intel Core i5 processor, 4GB of RAM and $3.20 \mathrm{GHz}$ of processing power. All reported results are averaged from 5 runs of the protocol.

All the participating parties initially agree upon the ECC parameters required for the respective ECC algorithm. The ring topology among the parties is set up and each party knows its neighbor on ring. One party in a ring is randomly designated as Initiator.

The secure three party addition protocol is implemented with four different homomorphic algorithms based on ECC. Our test application successfully shows fully functional secure three party addition protocol over real network.

\subsection{Experimental Results}

We evaluate ECC based encryption schemes for secure multiparty addition based on two metrics viz. the computational cost and the communication cost. Computational cost is measured as time taken for computation and communication cost is measured as number of bytes exchanged over communication channel.

We perform experiments with different ECC parameter sizes viz. 112-bit, 160-bit and 256-bit. For EC-OU, the prime value is 341 bit long and for remaining encryption schemes, prime value is similar to the size of Elliptic Curve Parameter Size i.e. 112 bit or 160 bit or 256 bit.

Table 2 shows the cost for running secure three party addition protocol using four ECC based encryption schemes.

As shown in Table 2, EC-EG gives better performance among all in terms of computational cost. However, if we consider the communication cost, EC-OU gives better performance. The result for higher computational cost for EC-OU is that in EC-OU, prime number p,q are 341 bits long and for other schemes prime numbers are same as the size of EC parameter size. To get the fair comparison, we then took random parameter size as 341 bits for all the algorithms and measure the cost of running secure multiparty addition using 341-bit value. The results are shown in Table 3. 
Table 2. Results for Secure Multiparty Addition using various ECC based encryption schemes.

\begin{tabular}{ccccccc}
\hline \multirow{2}{*}{$\begin{array}{c}\text { Encryption } \\
\text { Scheme }\end{array}$} & \multicolumn{2}{c}{ 112-bit } & \multicolumn{2}{c}{ 160-bit } & \multicolumn{2}{c}{256 -bit } \\
\cline { 2 - 7 } & Time (Mili seconds) & Cost (Kilobytes) & Time (Mili seconds) & Cost (Kilobytes) & Time (Mili seconds) & Cost (Kilobytes) \\
\hline EC-OU & 316 & 1116 & 335 & 1759 & 399 & 2719 \\
$E C-E G$ & 254 & 1740 & 286 & 2766 & 322 & 4865 \\
$E C-N S$ & 303 & 4224 & 349 & 4906 & 374 & 7487 \\
$E C-P$ & 247 & 2437 & 285 & & 351 & 7123 \\
\hline
\end{tabular}

Table 3. Results for Secure Multiparty Addition using various ECC based encryption schemes keeping 341-bit primes for all schemes.

\begin{tabular}{ccccccc}
\hline \multirow{2}{*}{$\begin{array}{c}\text { Encryption } \\
\text { Scheme }\end{array}$} & \multicolumn{2}{c}{ 112-bit } & \multicolumn{2}{c}{ 160-bit } & \multicolumn{2}{c}{ 256-bit } \\
\cline { 2 - 7 } & Time (Mili seconds) & Cost (Kilobytes) & Time (Mili seconds) & Cost (Kilobytes) & Time (Mili seconds) & Cost (Kilobytes) \\
\hline EC-OU & 316 & 1116 & 335 & 1441 & 399 & 2719 \\
$E C-E G$ & 309 & 4849 & 321 & 5181 & 357 & 6474 \\
$E C-N S$ & 336 & 5538 & 382 & 6198 & 407 & 8124 \\
$E C-P$ & 326 & 5515 & 356 & 6504 & 383 & 8760 \\
\hline
\end{tabular}

Table 3 shows that keeping 341-bit prime value fixed for all schemes results in higher computational cost for the encryption schemes other than EC-OU. In these experiments, we found that EC-OU and EC-EG takes lesser time where as EC-OU takes lesser space which is reasonably lesser than the other three schemes. Results for computational and communication cost are shown in Figures 2 and 3 respectively for varying length of primes.

Results for computational and communication cost for 341-bit fixed length prime are shown in Figures 4 and 5 respectively. As shown in Figures 4 and 5, if we consider both computational and communication cost, EC-OU perform better than all algorithms selected for evaluation.

\section{Conclusion}

In this paper, we propose a novel approach to securing multiparty computation using elliptic curve cryptography. We empirically evaluated various ECC based homomorphic encryption schemes for our proposed protocol. We demonstrate that EC-OU algorithm performs better among four selected algorithms. Our secure multiparty addition protocol achieves better efficiency in terms of communication cost as compared to corresponding secret sharing based approach and hence is scalable with respect to a number of parties. In addition, we highlighted various applications such as privacy preserving data mining as the candidate applications for our proposed approaches.

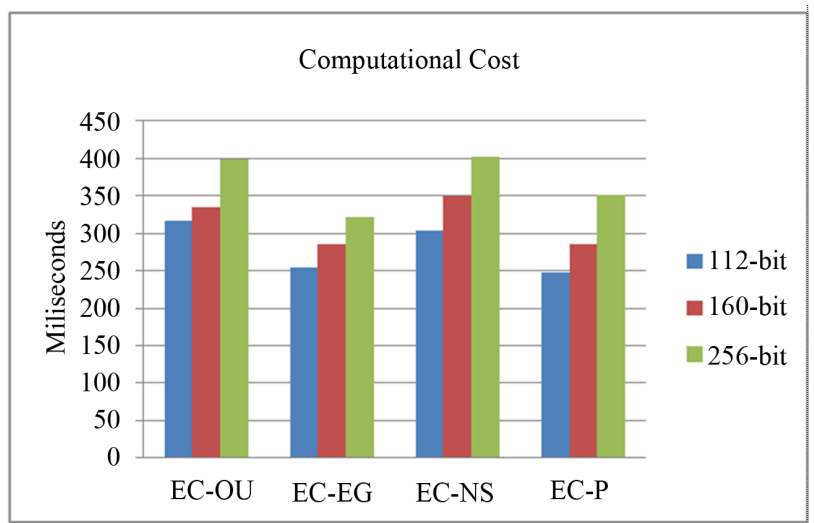

Figure 2. Comparison of Computatio nal cost for various ECC schemes for varying length of prime.

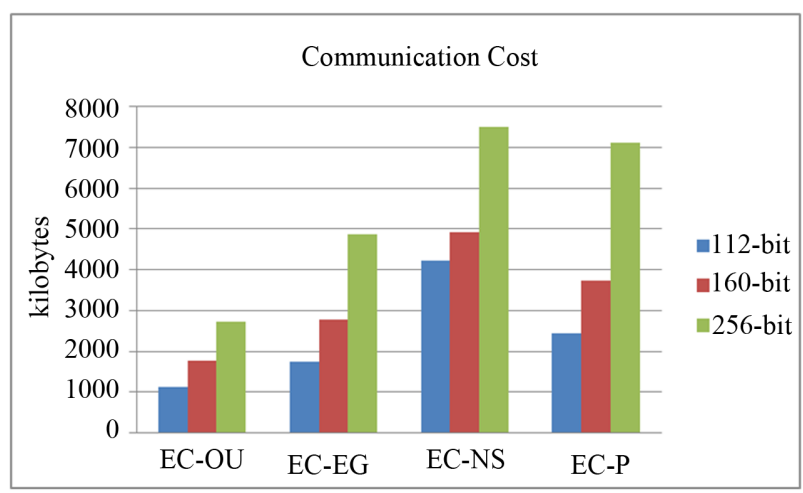

Figure 3. Comparison of Communication cost for various ECC schemes for varying length of prime. 


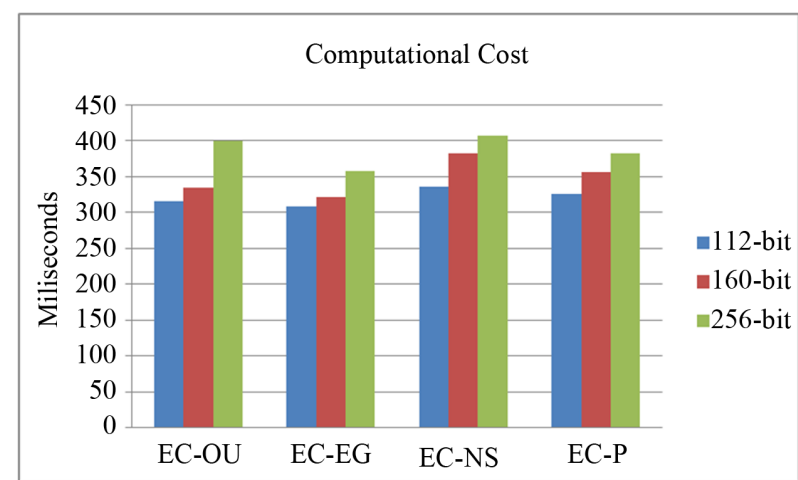

Figure 4. Comparison of Computational cost for various ECC schemes for 341-bit prime.

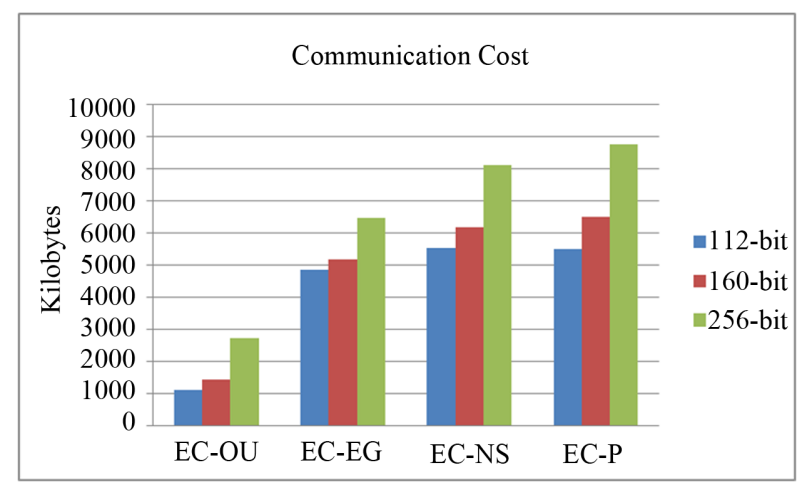

Figure 5. Comparison of Communication cost for various ECC schemes for 341-bit prime.

Our future includes incorporating EC-OU based secure multiparty computation in privacy preserving data mining application.

\section{REFERENCES}

[1] O. Goldreich, “The Foundations of Cryptography,” Vol. 2. Cambridge Univ. Press, Cambridge, 2004.

[2] Y. Lindell and B. Pinkas, "Secure Multiparty Computation for Privacy-Preserving Data Mining," Journal of Privacy and Confidentiality, Vol. 1, No. 1, 2009, pp. 59-98.

[3] M. Rabin, "How to Exchange Secrets by Oblivious Transfer,” Technical Report Tech. Memo TR-81, Aiken Computation Laboratory, 1981.

[4] D. Josep Ferrer, "A new privacy homomorphism and applications,” Information Processing Letters, Vol. 60, No. 5, 1996, pp. 277-282. http://dx.doi.org/10.1016/S0020-0190(96)00170-6

[5] A. Shamir, "How to Share a Secret," Communication of the ACM, Vol. 22, No. 11, 1979, pp. 612-613. http://dx.doi.org/10.1145/359168.359176

[6] T. B. Pedersen, Y. Saygin and E. Savas, "Secret Sharing vs. Encryption-Based Techniques for Privacy Preserving Data Mining," UNECE/Eurostat Work Session on SDC, 2007.

[7] S. Patel, S. Garasia and D. Jinwala, “An Efficient Ap- proach for Privacy Preserving Distributed K-Means Clustering using Shamir's Secret Sharing Scheme,” In: T. Dimitrakos, R. Moona and D. Patel, Eds., Trust Management VI, IFIP Advances in Information and Communication Technology, Vol. 347, Springer, Boston, 2012, pp. 129-144.

[8] G. Jagannathan and R. N. Wright, "Privacy-Preserving Distributed k-Means Clustering over Arbitrarily Partitioned Data,” KDD, ACM Press, 2005, pp. 593-599.

[9] S. Jha, L. Kruger and P. McDaniel, "Privacy Preserving Clustering," 10th European Symposium on Research in Computer Security, 2005, pp. 397-417.

[10] N. Koblitz, "Elliptic Curve Cryptosystems," Mathematics of Computation, Vol. 48, 1987, pp. 203-209. http://dx.doi.org/10.1090/S0025-5718-1987-0866109-5

[11] V. S. Miller, "Use of Elliptic Curve in Cryptography," In: Proceedings of Advances in Cryptology (CRYPTO'85), Springer Verlag, 1986, pp. 417-426.

[12] Certicom Research, "Standards for Efficient Cryptography_SEC 1: Elliptic Curve Cryptography,” 2009.

[13] A. C. Patel, U. P. Rao and D. R. Patel, "Privacy Preserving Association Rules in Unsecured Distributed Environment Using Elliptic Curve Cryptography,” Proceedings of International Conference on Computing Communication \& Networking Technologies (ICCCNT), 2012, pp. 1-5.

[14] M. Rajalakshmi and T. Purusothaman, "Privacy Preserving Distributed Data Mining using Randomized Site Selection,” European Journal of Scientific Research, Vol. 64, No. 4, 2011, pp. 610-624.

[15] A. C. Yao, "Protocols for Secure Computations," Proceedings of the 23rd Annual IEEE Symposium on Foundations of Computer Science, 1982, pp. 160-164.

[16] O. Goldreich, S. Micali and A. Wigderson, "How to Play any Mental Game," Proceedings of the 19th Annual ACM Symposium on Theory of Computing, 1987, pp. 218-229.

[17] B. Pinkas, "Privacy Preserving Data Mining," Journal of Cryptology, Vol. 50, No. 3, 2002, pp. 177-206. http://dx.doi.org/10.1007/s00145-001-0019-2

[18] B. Pinkas, "Cryptographic Techniques for Privacy-Preserving Data Mining," SIGKDD Explorations Newsletter, Vol. 4, No. 2, 2002, pp. 12-19. http://doi.acm.org/10.1145/772862.772865.

[19] W. L. Du and M. J. Atallah, "Privacy-Preserving Cooperative Scientific Computations," 14th IEEE Computer Security Foundations Workshop, Nova Scotia, 11-13 June 2001, pp. 273-282.

[20] W. L. Du and M. J. Atallah, "Privacy-Preserving Statistical Analysis," Proceedings of the 17th Annual Computer Security Applications Conference, New Orleans, 10-14 December 2001, pp. 102-110.

[21] W. L. Du and M. J. Atallah, "Protocols for Secure Remote Database Access with Approximate Matching," 7th ACM Conference on Computer and Communications Security (ACMCCS 2000), The First Workshop on Security and Privacy in E-Commerce, Athens, 1-4 November 2000, pp. 87-111.

[22] Y. Lindell and B. Pinkas, "Privacy Preserving Data Min- 
ing,” In Advances in Cryptology-Crypto2000, Lecture Notes in Computer Science, volume 1880, 2000.

[23] J. Vaidya and C. Clifton, "Privacy-Preserving k-Means Clustering over Vertically Partitioned Data," Proceedings of 9th ACM SIGKDD International Conference on Knowledge Discovery and Data Mining, ACM Press, 2003, pp. 205-216.

[24] J. Vaidya and C. Clifton, "Privacy Preserving Association Rule Mining in Vertically Partitioned Data," 8th ACM SIGKDD International Conference on Knowledge Discovery and Data Mining, 2002, pp. 639-644.

[25] S. Jha, L. Kruger and P. McDaniel, "Privacy Preserving Clustering," Proceedings of 10th European Symposium on Research in Computer Security, 2005, pp. 397-417.

[26] D. Naccache and J. Stern, “A New Public Key Crypto- system Based on Higher Residues,” ACM Conference on Computer and Communications Security, 1998, pp. 5966.

[27] T. Okamotoand S. Uchiyama, "A New Public-key Cryptosystem as Secure as Factoring,” EUROCRYPT, 1998, pp. 308-318.

[28] P. Paillier, "Public-Key Cryptosystems Based on Composite Degree Residuosity Classes,” EUROCRYPT, 1999, pp. 223-238.

[29] T. ElGamal, "A Public Key Cryptosystem and a Signature Scheme Based on Discrete Logarithms," CRYPTO, IT, Vol. 31, No. 4, 1985, pp. 469-472.

[30] P. Paillier, "Trapdooring Discrete Logarithms on Elliptic Curves over Rings,” ASIACRYPT, 2000, pp. 573-584. 